\title{
Optoelectronic system for phase array antenna beam steering
}

\author{
E. Sędek ${ }^{1}$, Z. Bielecki ${ }^{2}$, M. Muszkowski ${ }^{1}$, W. Kołosowski ${ }^{1}$, \\ G. Różański ${ }^{2} \& \mathrm{M}$. Wnuk ${ }^{2}$ \\ ${ }^{I}$ Telecommunications Research Institute (PIT), Poland \\ ${ }^{2}$ Military University of Technology (WAT), Poland
}

\begin{abstract}
This paper presents a system for optoelectronic beam steering of a phase antenna array. The microwave signal from an RF generator controlled by a pulse generator is transmitted to the electrical input of an optical elevation control unit, which generates $\mathrm{N}$ microwave signals with independent amplitude and phase. The control unit utilizes an optical wave from a laser. All outputs of those control units are connected to inputs of $\mathrm{N}$ optical azimuth control units, which provide $\mathrm{M}$ linear element antennas control. Those units utilize an optical wave from a second laser. This way an $\mathrm{M}$ x N element of a planar antenna control is provided. The presented method is compared with the classical and electronic method, which consists of multi-bits microwave phase shifters for producing an electronic scanning effect. On the basis of this concept we realized 16-element linear antenna array printed on a dielectric substrate fully controlled by an optoelectronic system. A tuned wavelength laser in the range 1520-1600 nm and $10 \mathrm{~mW}$ optical output power has been used as an optical signal source. The optical signal is modulated by a microwave signal. The applied modulator operates in the third optical window, allowing optical signal modulation to $10 \mathrm{GHz}$. Very high resolution and an excellent accuracy of the antenna beam positioning can be achieved. The optimal technique depends on the number of antenna elements, which implicates beam width. The presented method is preferred for very narrow antenna beams.
\end{abstract}

Keywords: optoelectronic system, phase array antenna, beam steering. 


\section{Introduction}

Active phase array antennas [1,2] have played an ever more important role in modern radar systems. This direction of antenna techniques has been possible due to electronic space scanning within a wide range without the need for mechanical rotation of the antenna as well as free options in the generated beam spatial characteristic shaping, achieved through relevant control of numerous transmission elements. The microwave control signals distribution for traditional systems take advantage of shared axis systems and metal wave-guides of considerable size; all these - due to a large number of active elements in an array antenna - make impossible a simple delivery of control signals and reception of output microwave signals. The rapid development of optoelectronic techniques, in particular development of numerous modern optic elements, used for fiber optic transmissions and optic signals processing, provide a practical opportunity to solve these problems [3-5].

\section{Electronic scanning of phase array antenna beam}

Active phased array antennas are composed of the matrix of independent microwave transmitting-receiving elements (TR-modules) to which separate control signals are being supplied by means of the independent regulated phase shifter systems or through delaying lines. The proper positioning in space of the radiating elements, as well as proper power supply, shape the resultant field distribution, making it possible to achieve the desired direction characteristic [6]. The generated beam spatial characteristics and propagation direction are controlled electronically, by changing an amplitude and phase of the elementary signals distributed to the individual antenna elements. The beam steering accuracy and spatial characteristic distortions depend upon the number of the applied antenna transmitting elements and accuracy in defining the phase delays and microwave signals power. Phased array antenna beam steering $[7,8]$ is based on supplying antenna radiating elements by microwave signals with gradually increasing phases. In the case of one-dimensional active antenna, the phase difference between antenna array consecutive elements required for shifting the antenna beam off an antenna axis by $\theta$ angle, can be presented as follows:

$$
\alpha=2 \pi \frac{d}{\lambda} \cos \theta
$$

where: $\lambda$ - microwave signal wavelength, $\mathrm{d}$ - distance between the radiating elements. Based on the dependence $\lambda=c / f$, where $c$ is the vacuum light speed, and $f$ - microwave signal frequency, we obtain:

$$
\alpha=2 \pi \cdot f \frac{d}{c} \cos \theta
$$


The above equation indicates that the phase of microwave signals connected to the antenna consecutive elements is dependent upon the microwave signal frequency. Thus, the antenna operational frequency change results in the beam propagation direction change; in turn, this indicates that varying frequencies are being positioned in different directions. This phenomenon is known in the literature as beam squinting. This disadvantage limits the antenna operation to monochromatic signals, thus disabling wide band systems implementation. Substituting in eqn (2), the dependence $\alpha=2 \pi \cdot f \cdot t$, where $t$ is a time needed for the wave to change phase by $\alpha$ and transforming the equation, we arrive at a simple dependence for the delay time of the microwave signal distributed to the antenna consecutive elements to propagate the beam under the $\theta$ angle:

$$
t=\frac{d}{c} \cos \theta
$$

The above equation shows that the signal delay time between the consecutive antenna elements - as opposite to the phase - does not depend on frequency, implicating a possibility of wide beam operation. Thus, the phase - array - in this case - should be replaced with the time-array type of steering. This method of steering is defined in the literature as TTD (true-time delay).

\section{Optoelectronic units}

A block scheme of the optoelectronic unit for 2-dimensional control of an antenna beam is presented in Fig. 1.

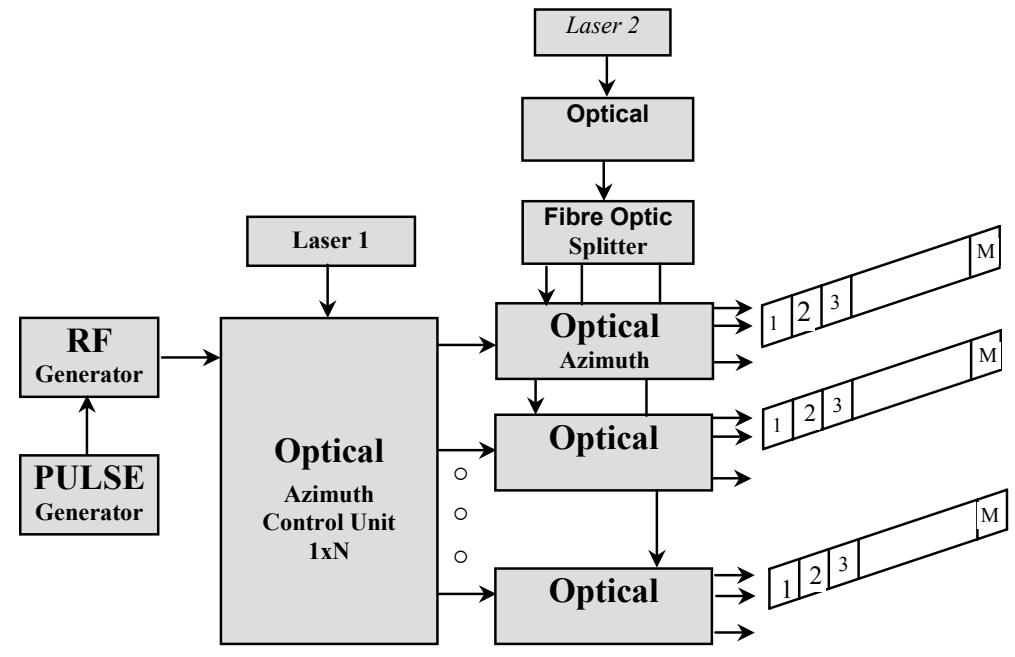

Figure 1: 2-dimensional optoelectronic control unit of antenna beam. 
Microwave signals from an RF generator controlled by a pulse generator are transmitted to an electrical input of an optical elevation control unit, which generates $\mathrm{N}$ microwave signals with independent amplitude and phase. The control unit utilizes an optical wave from a laser. All outputs of those control units are connected to inputs of $\mathrm{N}$ optical azimuth control units, which provide $\mathrm{M}$ linear element antennas control. Those units utilize an optical wave from a second laser. This way an MxN element of planar antenna control is provided. A block scheme of a 1-dimensional optoelectronic control unit of an antenna beam is presented in Fig. 2.

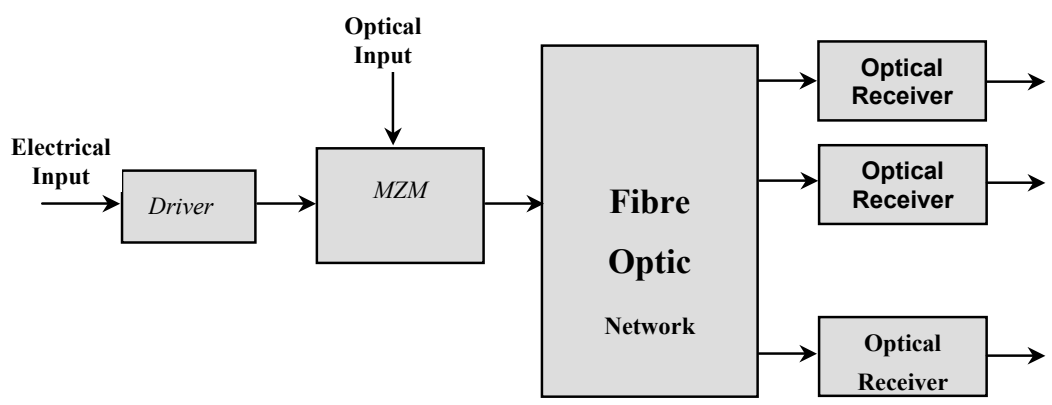

Figure 2: 1-dimensional optoelectronic control unit of antenna beam.

Optical input of the unit is supplied by the optical carrier signal from a laser. Electrical input is supplied by a microwave signal from the RF generator. A Mach-Zender optical modulator has been used to perform modulation of the optical carrier wave by a microwave signal. The modulated signal is routed to optical receivers via a fibre optic network, where the optical signal is split on $\mathrm{N}$ or $\mathrm{M}$ outputs and amplitude and phase control of each signal is provided. Optical to electrical conversion is done by optical receivers. There are many different optoelectronic approaches to arrange fibre optic network to amplitude and phase control, which can be divided into coherent and non-coherent systems. A coherent approach is based on optical signal phase modulation using coherent detection. Non-coherent systems are based on different lengths of optical path applications to provide various delay times of optical signals. In this case phase shift is proportional to length of optical fibre as follows:

$$
\Delta \alpha=2 \pi \cdot n \cdot f \frac{L}{c}
$$

where: $n$ - refractive index coefficient, $f$ - microwave signal frequency, L fibre optic length, $c$ - light velocity. This means that the optical signal phase change required to generate a microwave beam from a planar antenna propagated in the required direction needs to use a large number of different length fibre optic lines. For each beam position another set of different lengths of fibre optic 
is required, then we need $\mathrm{M} \times \mathrm{N} \times \mathrm{R}$ fibres, where $\mathrm{R}$ - number of beam position. Thus systems like this are very large and complicated.

Generally large numbers of different optic techniques were applied to achieve a variable phase shift. Most popular applications are based on: optical switches, piezo-electric crystals, liquid and aqustooptic crystals, high dispersion fibres and bragg gratings.

Two types of fibre optic techniques based on binary fibre optic delay line and dispersion delay line will be considered and compared to classic microwave phase shifters.

The microwave beam position generated from a linear antenna can be described as follows:

$$
\theta=\arccos \left(\frac{c}{d} \frac{\alpha}{2 \pi \cdot f}\right)
$$

where $\alpha$ is the phase difference of signals supplying antenna neighbour's elements, and $\mathbf{d}$ is a space between antenna elements. Concerning classical microwave systems applying 5-bit microwave phase shifters, the maximum phase resolution is 11.25 degrees. Then the maximum beam position angle is 4.77 degrees. Using an optic delay line with optical switches and different lengths of fibres the maximum resolution of fibre length is equal to $5 \mathrm{~mm}$ so then the maximum beam position angle is 1.7 degrees for the $5 \mathrm{GHz}$ microwave signal frequency. Much higher resolution can be achieved using a dispersion delay line. In this case we need to apply a variable wavelength laser with $0.1 \mathrm{~nm}$ resolution. Then the maximum phase resolution is equal to 0.25 degrees, and thus the maximum beam position angle is equal to 0.09 degrees for the $5 \mathrm{GHz}$ microwave signal frequency.

The scheme of the control system applies a material dispersion phenomenon in single-mode optical fibres for a 16-element linear antenna as presented in Fig. 3. A tuned wavelength laser in the range $1520 \div 1600 \mathrm{~nm}$ and an $10 \mathrm{~mW}$ optical output power has been used as an optic signal source. The optic signal is routed to $n$ electrooptic modulator.

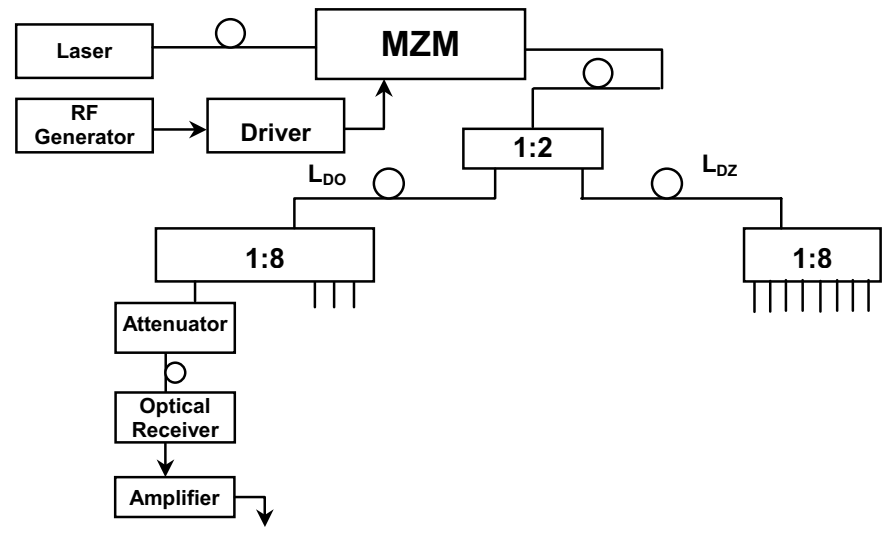

Figure 3: Antenna control system structure. 
The applied modulator operates in a third optic window, allowing for $10 \mathrm{GHz}$ optical signal modulation. A specialised microwave amplifier has been applied as a modulator driver.

The microwave input signal, following relevant amplification in a driver, is routed to the modulator electric input. Thus, an optic signal on the modulator output is amplitude modulated with an envelope according to the control microwave signal from the RF generator. Next, the modulated optic signal is distributed to a binary tree introducing the relevant microwave signal delays on individual outputs of the system. Optical signals are attenuated in fibre optic attenuators to obtain the required power distribution. Next, they are routed to the inputs of 16 optical receivers, performing optic electric signal conversion and finally distributed to the microwave amplifiers, thus additional amplifcation of $22 \mathrm{~dB}$ is performed. The antenna beam control system requires selection of high dispersion fibre optic length $\mathrm{L}_{\mathrm{D}}$ and zero dispersion fibre optic length $\mathrm{L}_{\mathrm{ZD}}$, according to the operation laser wavelength range to obtain the desired antenna beam scanning range.

\section{Control system measurements}

Detail measurements of the developed system are based on measurements of the output signal power distribution and microwave signal delay between a modulating signal and 16 outputs signals of the control system. The measurements have been done on a microwave network analyser HP8720B. The power and phase of the microwave signal have been measured. The phase was converted into a time delay for a signal frequency equal to $5 \mathrm{GHz}$. The antenna elevation characteristics have been calculated on the basis of the obtained results. Antenna beam scanning in the range of $0^{\circ} \div 45^{\circ}$ has been achieved.

In figure 4 high compatibility between the position angle of a 16-element linear antenna characteristic in theory (black curve) and measurement (red curve) for a fibre optic dispersion delay line application can be observed.

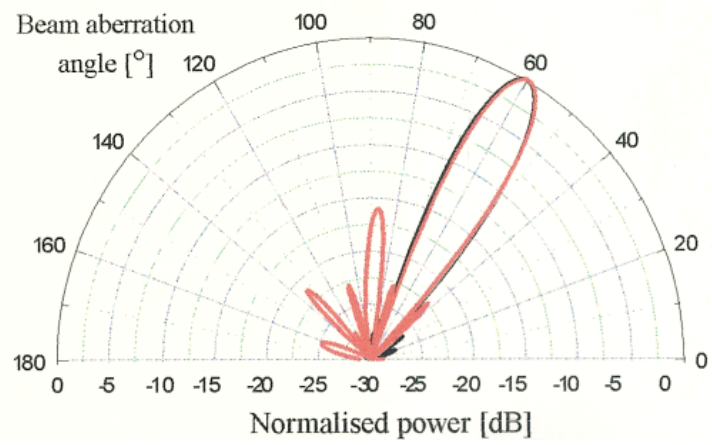

Figure 4: 16-element linear antenna characteristics for $30^{\circ}$ degree antenna beam position. 
Elevation characteristics of an antenna, calculated theoretically and based on measurements for a distribution of output power like $\cos ^{2} x+0.4$ are presented in Figs 5 and 6. Radiation characteristics of the 16-element antenna row, performed for a non-uniform signal power distribution at the control system outputs proves considerable compatibility with theoretical simulations. The developed system ensures an opportunity to control the beam propagation direction with an excellent accuracy equal to $0.1^{\circ}$.

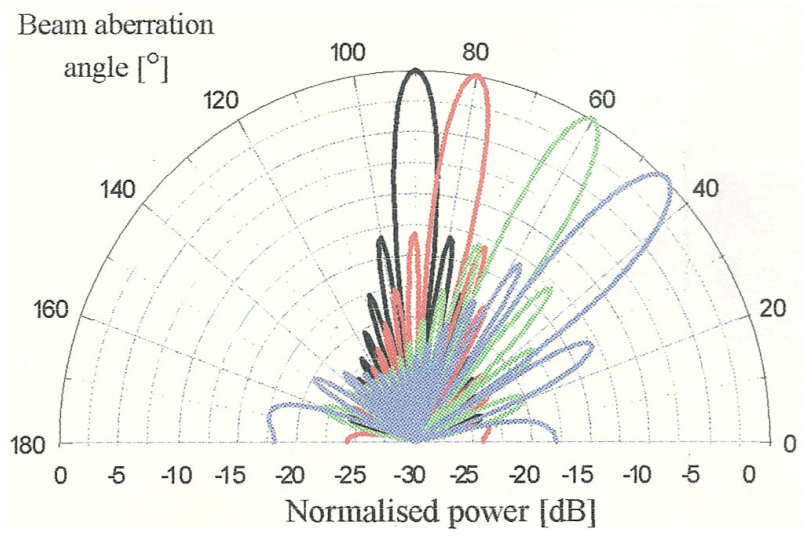

Figure 5: 16-element linear antenna characteristics for $0^{\circ}, 10^{\circ}, 30^{\circ}$ and $45^{\circ}$ degree antenna beam position (simulation).

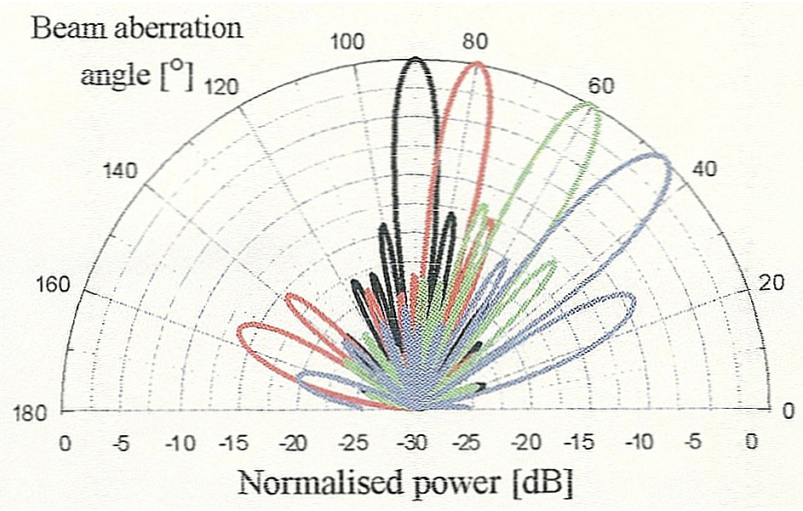

Figure 6: Antenna characteristics for $0^{\circ}, 10^{\circ}, 30^{\circ}$ and $45^{\circ}$ degree antenna beam position (measurements).

\section{Conclusion}

Using digital microwave phase shifters the calculated phase shift must be approximated according to phase shifter resolution, then the accuracy of beam 
positioning is equal to a few degrees. Application of optical weigh units in phase array beam forming provides full antenna characteristic control. Very high resolution and an excellent accuracy of the antenna beam positioning can be achieved. The optimal technique depends on the number of antenna elements, which implicates beam width. For very narrow beams the best solution is optical delay line application in weigh units providing high angle resolution and positioning accuracy.

\section{References}

[1] Mailloux R.J., Phased Array Antenna Handbook, Artch House Inc. 1994

[2] Dufrêne R., Sędek E., Kołosowski W., Wnuk M., Lisowski J., Muszkowski M., Array Antenna Direction Characteristics Shaping Optic and Electronic Methods, PIT Works No. 127, Warsaw 2001

[3] Zmuda H., Toughlian E.N., Photonic Aspect of Modern Radar, Artech House Inc. 1994

[4] Kumar A., Antenna Design with Fiber Optics, Artech House Inc. 1996

[5] Muszkowski M., Radar Signals Fiber Optic Transmission System, New Constructions and Technologies, Rościszów 1988

[6] Seeds A.J., Application of Opto-Electronic Techniques in Phased Array Antenna Beamforming, Microwave Photonic, Technical Digest, MWP 1997

[7] Frigyes I., Seeds A.J., Optically Generated True-Tine Delay in PhasedArray Antennas, IEEE Trans. Microwave theory \& Tech., vol. 43. p. 2378-2386, Sept. 1995

[8] Soref R., Optical Dispersion Techniques for Time-Delay Beam Steering, Appl. Opt. Vol. 31, No. 36, p. 7395-7397, Dec. 1992 